\title{
Title: Mass seasonal bioflows of high-flying insect migrants
}

\author{
Authors: Gao Hu, ${ }^{1,2,3 *}$ Ka S. Lim, ${ }^{2}$ Nir Horvitz, ${ }^{4}$ Suzanne J. Clark, ${ }^{2}$ Don R. Reynolds, ${ }^{5}$ Nir \\ Sapir, ${ }^{6}$ Jason W. Chapman ${ }^{2,3 *}$
}

\begin{abstract}
Affiliations:
${ }^{1}$ College of Plant Protection, Nanjing Agricultural University, Nanjing, China.

${ }^{2}$ Rothamsted Research, Harpenden, Herts, UK.

${ }^{3}$ Centre for Ecology and Conservation, and Environment and Sustainability Institute, University of Exeter, Penryn, Cornwall, UK.

${ }^{4}$ Movement Ecology Laboratory, Department of Ecology, Evolution \& Behavior, The Hebrew University, Jerusalem, Israel.

${ }^{5}$ Natural Resources Institute, University of Greenwich, Chatham, Kent, UK.

${ }^{6}$ Animal Flight Laboratory, Department of Evolutionary \& Environmental Biology, University of Haifa, Haifa, Israel.
\end{abstract}

*Corresponding author. Email: hugao@njau.edu.cn (G.H.); j.chapman2@exeter.ac.uk (J.C.) 


\begin{abstract}
Migrating animals impact ecosystems directly via influxes of predators, prey and competitors, and indirectly by vectoring nutrients, energy and pathogens. While linkages between vertebrate movements and ecosystem processes have been established, the effects of mass insect 'bioflows' are undescribed. We quantified biomass flux over southern UK for high-flying (>150 m) insects, and show that $\sim 3.5$ trillion insects (3200 tons of biomass) migrate above the region annually. These flows are not randomly directed in insects larger than $10 \mathrm{mg}$, which exploit seasonally-beneficial tailwinds. Large seasonal differences in the southward versus northward transfer of biomass occur in some years, although flows were balanced over the 10-year period. Our long-term study reveals a major transport process with implications for ecosystem services, processes and biogeochemistry.
\end{abstract}

One Sentence Summary: Enormous long-distance atmospheric transport of insect biomass occurs in seasonally-predictable directions 
Main Text: Latitudinal migrations of vast numbers of flying insects, birds and bats (1-7) lead to huge seasonal exchanges of biomass and nutrients across the Earth's surface (8-11). Because many migrant species (particularly insects) are extremely abundant $(1,5)$, seasonal migrations may profoundly affect communities through predation and competition while transferring enormous quantities of energy, nutrients, propagules, pathogens and parasites between regions, with substantial effects upon essential ecosystem services, processes and biogeochemistry $(8-11)$, and ultimately ecosystem function.

Latitudinal bird migrations are well characterized; e.g. 2.1 billion passerines migrate annually between Europe and Africa (2), integrating multi-sensory navigational information (12), exploiting favorable winds and adopting adaptive flight behaviors (13). By comparison, even though insect migration surpasses all other aerial migratory phenomena in terms of sheer abundance (1), latitudinal insect migration is largely unquantified, in particular for the majority of species that migrate hundreds of meters above the ground (5). Specialized radar techniques are required to study these high-flying insect migrants as they are too small to carry transmitters or be observed by any other means (14). Until now, radar studies have been aimed almost exclusively at quantifying migrations of relatively few nocturnal species of agricultural pests (3), and no study has yet quantified the hugely abundant and ecologically-important daytime insect migrants.

We quantified annual abundance and biomass of three size categories of diurnal and nocturnal insects migrating above an area of $\sim 70,000 \mathrm{~km}^{2}$ of the southern UK (Fig. 1A), between $150-1200 \mathrm{~m}$ above ground level (agl) (Fig. 1B), from 2000-2009 (15). Abundance and biomass values for medium (10-70 $\mathrm{mg}$ ) and large insects $(70-500 \mathrm{mg}$ ) (referred to collectively as 'larger insects') were calculated from measurements of $>1.8$ million individuals (table S1) detected by Vertical-Looking entomological Radars (VLRs) located in southern UK (Fig. 1A). The VLRs provide a range of information - including body mass, flight altitude, aerial density, displacement speed, displacement direction, and flight heading - for all individual insects of $>10 \mathrm{mg}$ body mass that fly through the vertically-pointing beam within the altitude range of 150-1200 m agl (14). Annual abundance and biomass values for larger insects migrating over the study area were extrapolated from the aerial densities and body masses recorded above the VLR 
locations (15). The third size category, small insects $(<10 \mathrm{mg})$, are not sampled by VLRs, and so abundance and biomass data were calculated from aerial netting samples (16) taken $200 \mathrm{~m}$ agl near one of the radars (Fig. 1A) and extrapolated to the study area (15). Larger diurnal migrants are predominantly beneficial species, including hoverflies, ladybeetles, carabid beetles, and butterflies (14-17), and the most abundant small day-fliers are cereal aphids (16). The commonest larger nocturnal insects are lacewings and noctuid moths $(14,16)$, while Diptera comprise the majority of the small nocturnal insects $(16)$.

An annual mean of 3.37 trillion insects (range: $1.92-5.01 \times 10^{12}$; Fig. 1C and table S2) migrated high above the study region, comprising 3200 tons of biomass (fig. S1 and table S3), of which >70\% occurred during daytime (Fig. 1C and table S2). Numerically, >99\% of individuals were small insects, yet while the 15 billion medium and 1.5 billion large insects comprised only $0.4 \%$ and $0.05 \%$ of the annual abundance (table S2), they accounted for a substantial proportion of the biomass: 12\% (380 tons) and 7\% (225 tons) respectively (table $\mathrm{S} 3$ ).

By analyzing 1,320 daytime 'mass migrations' (15) involving 1.25 million VLR-detected insects, and 898 nocturnal mass migrations involving 126,000 insects (table S1), we characterized migration directions of the larger insects during 'spring' (May-June), 'summer' (July) and 'fall' (AugustSeptember) (fig. S2 and table S4). Although high-altitude winds blew consistently towards the northeast/east in all three seasons (Rayleigh tests; daytime: spring, $60^{\circ}$; summer, $66^{\circ}$; fall, $84^{\circ}$; night-time: spring, $69^{\circ}$; summer, $81^{\circ}$; fall, $101^{\circ}$; Fig. $2 \mathrm{~A}$ and table S5), mass migrations of larger insects did not simply move with the prevailing south-westerly winds. During the spring, mass migrations were consistently towards the north (Rayleigh tests; daytime: medium, $333^{\circ}$; large, $329^{\circ}$; night-time: medium, $349^{\circ}$; large, $349^{\circ}$; Fig. 2A), indicating that migration occurred on winds with a significantly more southerly component than prevailing winds (Watson-Wheeler tests; $P<0.0001$ in all cases; table S5). Summer mass migrations were randomly directed (Rayleigh tests; $P>0.05$ in all cases; Fig. 2 A and table S5), indicating an absence of wind selectivity. By contrast, fall mass migrations were consistently directed towards the south (Rayleigh tests; daytime: medium, $174^{\circ}$; large, $159^{\circ}$; night-time: medium, $181^{\circ}$; large, $180^{\circ}$; Fig. $2 \mathrm{~A}$ ), indicating active selection of winds with a significantly more northerly 
component than the prevailing winds (Watson-Wheeler tests; $P<0.0001$ in all cases; table S5). These relationships indicate preferred movement directions during the spring and fall, and selection of days and nights with favorably-directed tailwinds. Seasonally-beneficial migration directions have been previously reported in a few species of large insects, notably pest noctuid moths $(3,14,18)$, but our findings demonstrate the ubiquity of such movements among a diverse array of insect migrants for the first time. Since small insects fall below the VLR's detection threshold (14) and thus their tracks cannot be directly measured, we used aphid migration intensity as representative of all small insect migration (fig. S3) by analyzing wind directions associated with mass migrations of aphids (15). We found that aphid migration directions closely match prevailing wind directions, i.e. towards the north-east in all seasons (fig. S4), and therefore conclude that these small insects do not have mechanisms for selecting seasonally-beneficial winds.

The greatest amount of variation in the aerial density of diurnal migrants was explained by surface meteorological conditions associated with fine weather (table S6): migration intensity was greatest on warm days (fig. S5A), with moderate to high surface heat flux (fig. S5B), and low surface wind speeds (fig. S5C). However, the strong relationship with fine weather does not explain the directed movements of the larger insects in spring and fall. Models indicated that during these seasons (but not summer), surface wind direction was also correlated with migration intensity, with high densities associated with southerly winds in the spring and northerly winds in the fall (table S6). Surface and high-altitude daytime wind directions were strongly correlated in all seasons (tests for T-linear association; $P<0.001$ in all cases; fig. S6), but not at night (18). Thus surface wind direction provides a reliable cue regarding the suitability of winds aloft for diurnal migrants at take-off, but not for nocturnal migrants which must employ other methods for assessing high-altitude wind direction (19). The ubiquity of tailwind selectivity in such a diverse group indicates that compass mechanisms must be universal in larger insect migrants.

If high-flying insects have a compass sense, one would predict that in addition to selecting a favorable tailwind, they would also orientate in the seasonally-beneficial direction, and thus actively contribute to their wind-assisted displacement. Such "common orientation" was indeed highly prevalent in the larger 
insects (table S1), and headings were close to tracks (fig. S7): northwards in the spring and southwards in the fall (table S7). The close correspondence between headings and tracks signifies that larger insects added much of their self-powered airspeed to the wind vector, and thus achieved rapid displacement speeds [10-16 m/s (36-58 km/h); Fig. 2B; table S7]. A flight duration of 4 hours could therefore result in transport over $>200 \mathrm{~km}$, and during spring and fall this transfer of biomass and nutrients occurs in predictable directions.

What are the implications of this high-altitude insect movement? Insect bodies are typically comprised of $10 \%$ nitrogen and $1 \%$ phosphorus by dry weight (20), and as such they represent a rich source of nutrients which can be limiting for plant productivity (11). The 3200 tons of biomass moving annually above our study region contains $\sim 100,000 \mathrm{~kg}$ of $\mathrm{N}$ and $10,000 \mathrm{~kg}$ of $\mathrm{P}$, representing $0.2 \%$ of the surface deposits of $\mathrm{N}$ and $0.6-4.7 \%$ of $\mathrm{P}$ from the atmosphere, comprising $5.78 \times 10^{12}$ Joules of energy (15). To put these seasonal movements in context, the annual airborne insect biomass $>150 \mathrm{~m}$ above the southern UK is 4.5 times greater than the 2.2 billion (700 tons) of bogong moths (Agrotis infusa) which migrate to the Australian Alps every summer (7, 9), 7.7 times greater than the 30 million songbird migrants $(415$ tons) which depart the UK for Africa each fall (table S8), and >40 times greater than the 150 million monarch butterflies (75 tons) which migrate between Eastern North America and Mexico (14).

If the spring and fall movements documented here perfectly counter-balance each other there would be no net annual exchange of energy and nutrients, and the principal consequences would be the exchange of genes, pathogens and parasites. Over the 10-year study we found that net northward spring movements of larger insects were almost exactly cancelled out by net southward fall movements; however, on an annual basis the net flux could be up to 200 tons greater in either direction (Fig. 3). Such insect movements represent an underappreciated mechanism for redistributing nutrients and energy, and if the densities observed over southern UK are extrapolated to the airspace above all continental landmasses, highaltitude diurnal insect migration represents the most important annual animal movement in terrestrial ecosystems, comparable to the most significant oceanic migrations (21). Given the worrying declines in many migrants (8), developing global surveillance techniques (6) for long-term observation and 
prediction of the impacts of mass aerial migrations at such macrosystem scales (22) should be a priority for ecologists.

\section{References and Notes:}

1. R. A. Holland, M. Wikelski, D. S. Wilcove, Science 313, 794-796 (2006).

2. S. Hahn, S. Bauer, S. Liechti, Oikos 118, 624-626 (2009).

3. J. W. Chapman et al., Science 327, 682-685 (2010).

4. J. W. Chapman et al., Proc. Natl. Acad. Sci. USA 109, 14924-14929 (2012).

5. J. W. Chapman, D. R. Reynolds, K. Wilson, Ecol. Lett. 18, 287-302 (2015).

6. R. Kays, M. C. Crofoot, W. Jetz, M Wikelski, Science 348, aaa2478 (2015).

7. E. Warrant et al., Front. Behav. Neurosci. 10, 77 (2016).

8. D. S. Wilcove, M. Wikelski, PLOS Biol. 6, e188 (2008).

9. K. Green, Austral Ecol. 36, 25 - 34 (2011).

10. S. Bauer, B. J. Hoye, Science 344, 1242552 (2014).

11. J. S. Landry, L. Parrott, Ecosphere 7, e01265 (2016).

12. H. Mouritsen, D. Heyers, O. Güntürkün, Annu. Rev. Physiol. 78, 10.1-10.22 (2016).

13. T. Alerstam et al., Proc. Biol. Sci. 278, 3074-3080 (2011).

14. V. A. Drake, D. R. Reynolds, Radar Entomology: Observing Insect Flight and Migration (CABI, Wallingford, UK, 2012).

15. See the supplementary materials and methods

16. J. W. Chapman, D. R. Reynolds, A. D. Smith, E. T. Smith, I. P. Woiwod, Bull. Entomol. Res. 94, 123-136 (2004).

17. C. Stefanescu et al., Ecography 36, 474-486 (2013).

18. J. W. Chapman et al., Curr. Biol. 18, 514-518 (2008).

19. J. W. Chapman et al., Curr. Biol. 25, R751-R752 (2015). 
20. J. J. Elser et al., Nature 408, 578-580 (2000).

21. Ø. Varpe, Ø. Fiksen, A. Slotte, Oecologia 146, 443-451 (2005).

22. J. F. Kelly, K. G. Horton, Global Ecol. Biogeogr. DOI: 10.1111/geb.12473 (2016).

Acknowledgments: G.H.'s visiting scholarship was funded by the China Scholarship Council. We acknowledge the support provided by COST - European Cooperation in Science and Technology through the Action ES1305 "ENRAM". The project was supported by BBSRC grant BB/J004286/1 to J.W.C. Data are available at Dryad at http://xxxxxx/xxxxxx/cccccc.

\section{Supplementary Materials:}

Materials and Methods

Figures S1-S8

Tables S1-S8

References (1-49) 


\section{Figure Legends}

Fig. 1. Monitoring migration intensity above the southern United Kingdom. (A) The intensity and direction of high-altitude insect migration through the atmosphere 150-1200 m above ground level (agl) was measured over a 70,000 $\mathrm{km}^{2}$ region of the southern UK (black circle) under continual surveillance from vertical-looking radars (VLR, left inset) at 3 locations (white dots); the aerial insect fauna was sampled by balloon-supported aerial netting at $200 \mathrm{~m} \mathrm{agl} \mathrm{(center-right} \mathrm{inset)} \mathrm{and} \mathrm{12-m} \mathrm{high} \mathrm{suction} \mathrm{traps}$ (bottom-right inset). (B) Vertical profiles of larger insect (>10 mg) migration intensity over the sampling range of the VLRs. (C) Annual totals of insects migrating above the study region, during daytime, dusk, and night, and combined for the whole 24 hours. Lines represent annual totals; in the box plots, the central bar represents the median, boxes represent the interquartile range (IQR), whiskers extend to observations within \pm 1.5 times the IQR and dots represent outliers.

Fig. 2. Migratory directions and speeds of high-flying larger insects. (A) Despite prevailing winds blowing towards the northeast in all seasons, migratory tracks and headings occurred predominantly in seasonally-beneficial directions in spring and fall, but were randomly directed in summer. Small black circles on the periphery of the circular histograms represent mean directions of individual mass migrations, and the color bar indicates the percentage of migrations in each $22.5^{\circ}$ bin. The bearing of the blue arrow indicates the overall mean direction, and arrow length represents the circular resultant length (r). (B) Migrants achieved fast displacement speeds. Solid black bars represent medians, dashed red lines represent means, boxes represent the IQR, whiskers extend to observations within \pm 1.5 times the IQR, and dots represent outliers. 
Fig. 3. Annual patterns of net directional migration. The net flow of biomass of larger insects above the study region, in spring (blue), fall (red), and the whole year (black). Negative values indicate a net southward movement, while positive values indicate a net northward movement. In the box plots, central bars represent median values, boxes represent the IQR, whiskers extend to observations within \pm 1.5 times the IQR, and dots represent outliers. 
Fig $1 \mathrm{~A}$

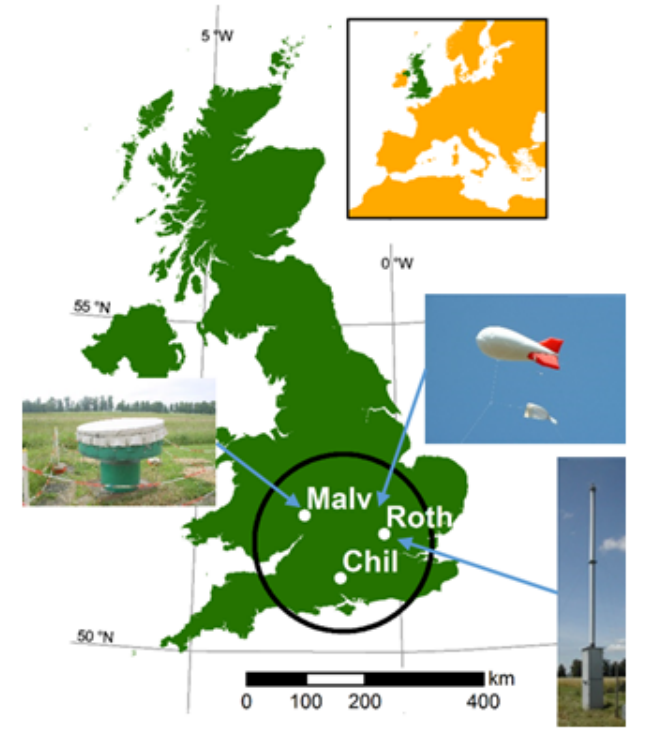

Fig 1B

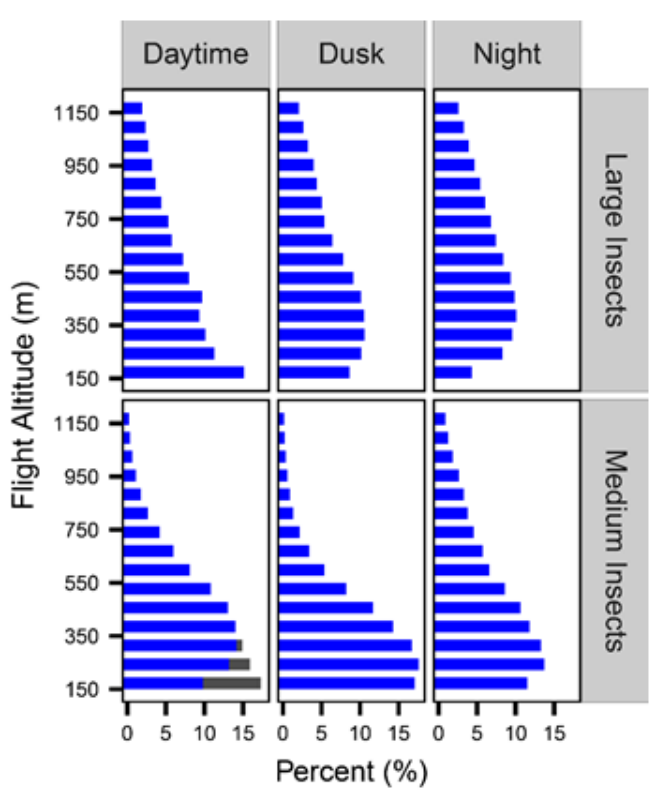


Fig $1 C$

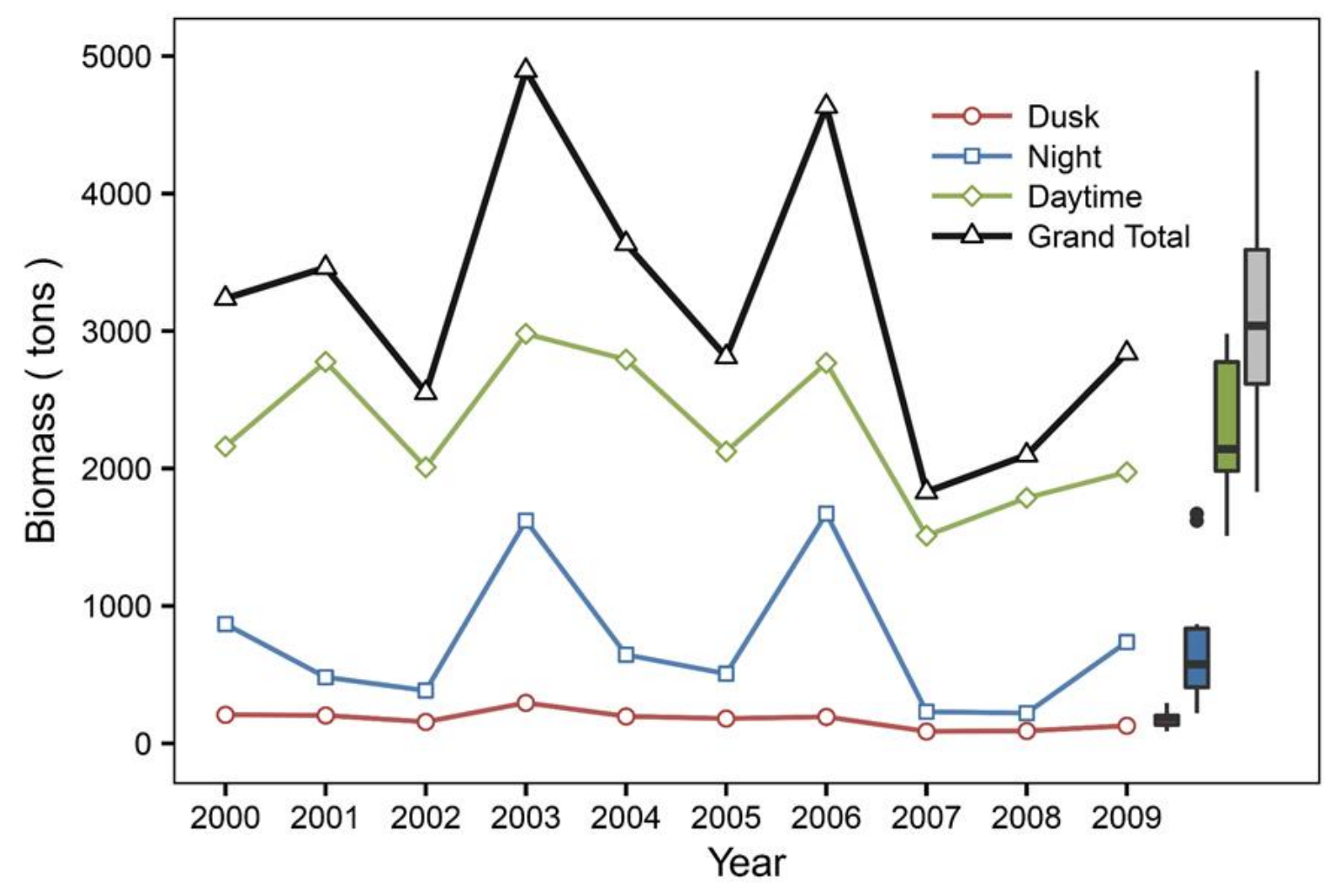


Fig 2A

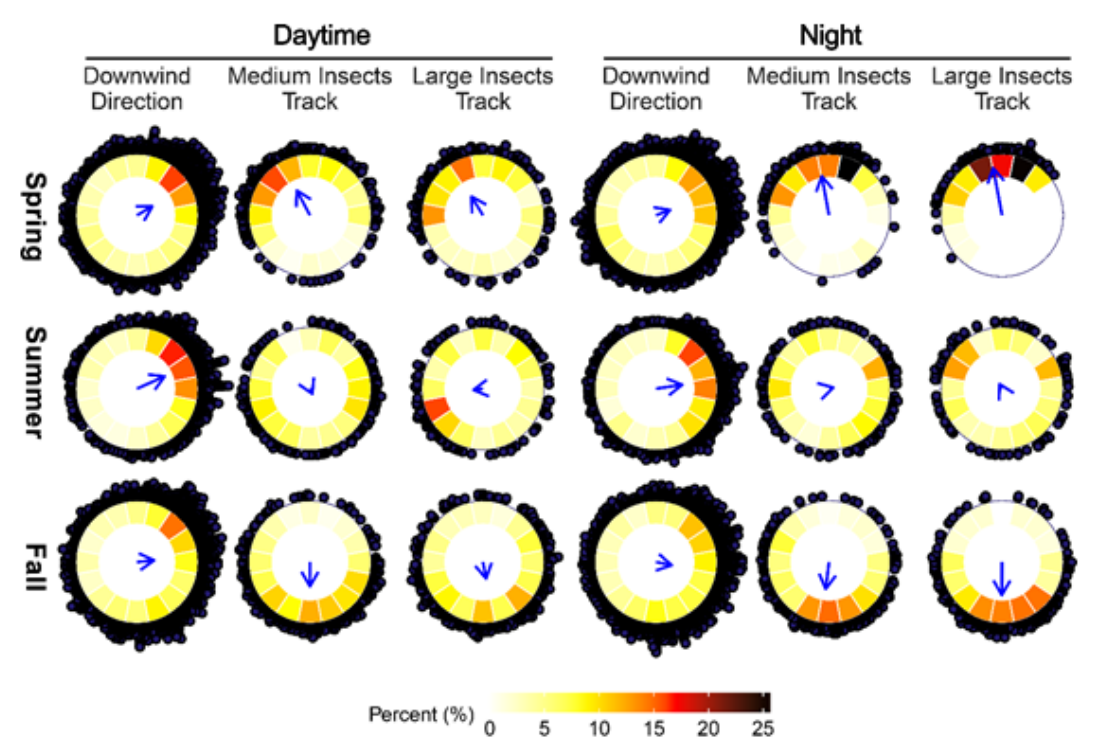

Fig 2B

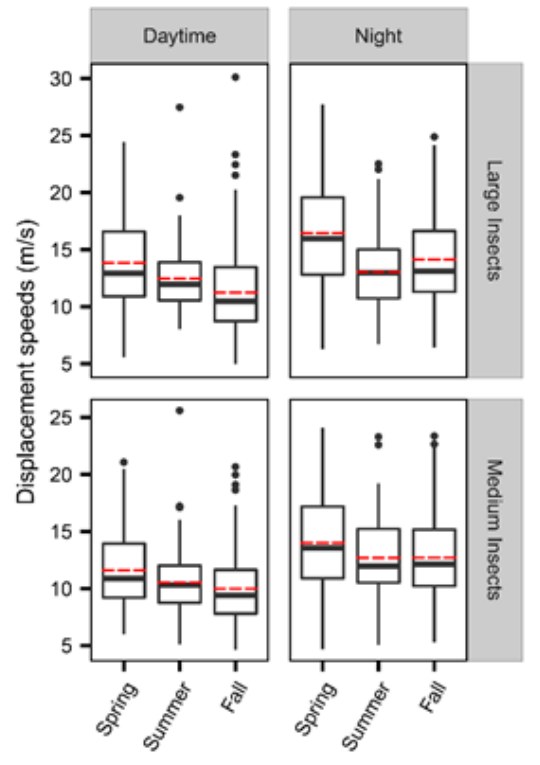


Fig 3

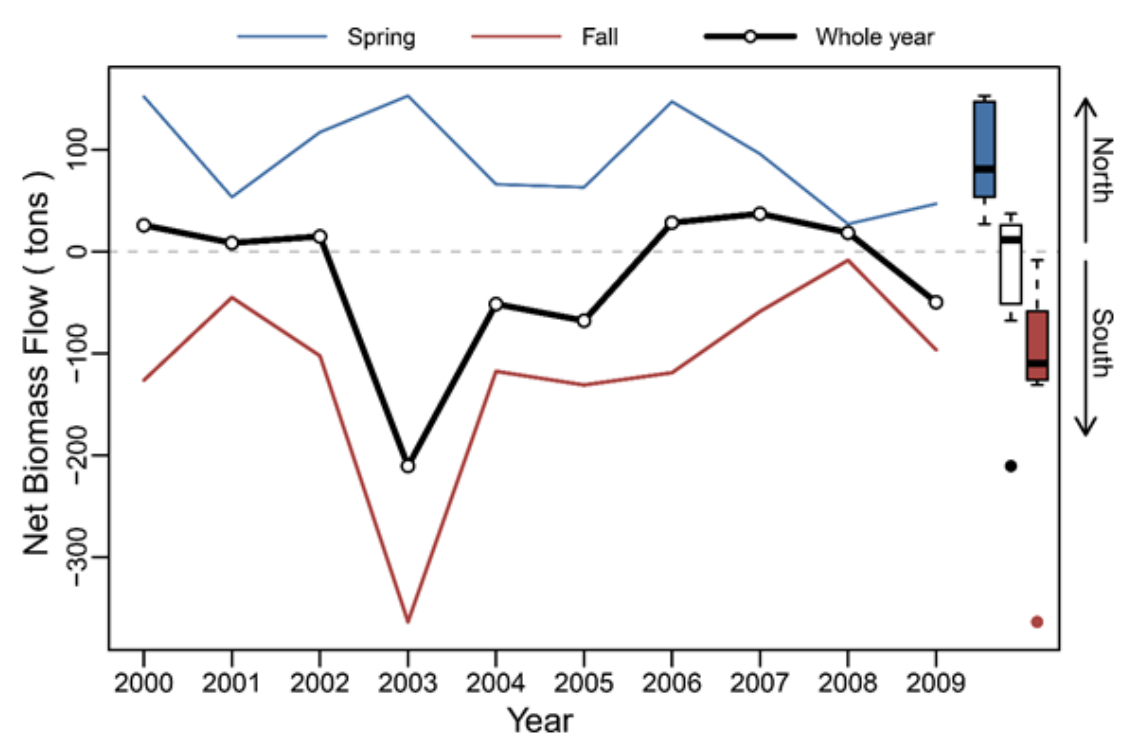

